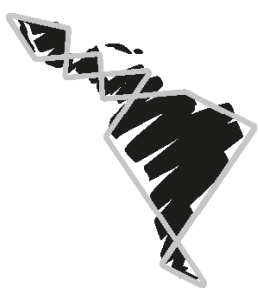

\title{
Variables incidentes en la trata de personas. El caso de mujeres adolescentes en el Estado de Chihuahua
}

\author{
Incidential Variables in Human \\ Trafficking. Adolescent Women's \\ Case in Chihuahua
}

\begin{abstract}
Variáveis que afetam o tráfico de pessoas. o caso das mulheres adolescentes no Estado de Chihuahua
\end{abstract}

\section{Martha Aurelia Dena Ornelas ${ }^{1}$}

\section{Resumen}

Este trabajo expone los hallazgos de una investigación delineada para buscar causas de la presencia de la trata de personas en mujeres adolescentes del Estado de Chihuahua. La investigación se aborda a partir del paradigma cualitativo desarrollado y construido partiendo de la exploración de 28 casos a profundidad para estudiar el fenómeno de la trata con un acercamiento de tipo inductivo mediante la aplicación de entrevistas semiestructuradas, análisis documental de expedientes jurídicos y seguimiento hemerográfico de notas de prensa. Se logran establecer con claridad cuatro variables incidentes para la victimización, y se dejan como líneas futuras de un estudio con mayor nivel de profundidad lo relativo al estatus migratorio y su incidencia con la trata sexual en el Estado de Chihuahua.

Palabras claves: Derechos Humanos, trata de personas, variables incidentes, mujeres adolescentes.

1 Adscripción institucional: Universidad Autónoma de Chihuahua. Docente adscrita al programa de maestría en Administración Pública de la Facultad de Ciencias Políticas y Sociales de la UACH, campus Ciudad Juárez. Licenciada en Derecho por la Universidad Autónoma de Ciudad Juárez. Maestra en Administración Pública en el programa de maestría de la Facultad de Ciencias Políticas y Sociales de la Universidad Autónoma de Chihuahua. Alumna del quinto semestre del programa de Doctorado en Ciencias Sociales de la Universidad Autónoma de Ciudad Juárez. Nacionalidad: Mexicana. 


\begin{abstract}
This document shows the findings of an investigation oriented to search the causes of human trafficking for sexual exploitation of adolescent women of the State of Chihuahua. This is a research developed and built from the exploration of 28 cases in depth to study the phenomenon of human trafficking with an inductive type approach through the application of semi-structured interviews, documental analysis of legal files and press releases. It successfully stablishes with clarity four incident variables for the victimization, having as future study lines along with a mayor level of in depth study, those linked to issues of migratory status and their relation with human trafficking in the State of Chihuahua.
\end{abstract}

Keywords: Human Rights, human trafficking, incident variables, teenage women.

\title{
Resumo:
}

Este artigo apresenta os resultados de uma investigação elaborada com a finalidade de encontrar as causas descritas sobre a presença do tráfico de mulheres adolescentes do Estado de Chihuahua. A pesquisa é abordada a partir do paradigma qualitativo, desenvolvido e construído com base na análise em profundidade de 28 casos. O objetivo foi estudar o fenómeno do tráfico com uma abordagem de tipo indutivo, através da aplicação de entrevistas semiestruturadas, análise documental dos registros legais e rastreamento hemerográfico das notícias dos jornais. A ideia é estabelecer de forma clara quatro variáveis importantes para vitimização, deixando para futuras linhas de estudo, com níveis altos para aprofundar o tema relacionado ao status de imigração e sua influencias ao tráfico sexual no Estado de Chihuahua.

Palavras-chave: Direitos humanos, tráfico de mulheres, variáveis importantes, mulheres adolescentes.

\section{Introducción}

La trata de personas tiene causas múltiples y complejas, esta se ha presentado en parte como un resultado de la desigualdad social, de la debilidad del Estado-nación, y de los avances tecnológicos que permiten comercializar con seres humanos, entre otros fenómenos. Independientemente de sus razones, esta actividad se ha convertido en una práctica delictiva de orden transnacional y se ubica en el tercer lugar entre las actividades ilícitas más lucrativas, está después del tráfico de armas y el narcotráfico. La Organización Internacional del Trabajo (OIT) estima que las ganancias anuales producidas por la trata de personas son alrededor de 32,000 millones de dólares (Estévez, 2011) y que 2.5 millones de personas en el mundo han sido objeto de trata, de las cuales un $43 \%$ son utilizadas para explotación sexual, un $32 \%$ en trabajo forzado y otro $25 \%$ son víctimas sujetas a ambas modalidades (Acharya, 2016). Estos datos permiten apreciar la magnitud del problema y dimensionar su manifestación más común, la explotación sexual, modalidad en la que se 
centra este estudio. El presente trabajo se centra en la búsqueda de causas que como variables extraídas desde diversas perspectivas teóricas se aplican en el análisis a profundidad de 28 casos de víctimas mujeres adolescentes que estuvieron sujetas a una de las mayores lesiones que reciben los seres humanos contra su dignidad. Lo anterior obliga a realizar una investigación como esta debido a la gama de derechos humanos que se transgreden al considerar el cuerpo del otro u otra como una mercancía susceptible de transacción comercial donde el objeto de la misma es el libre derecho al ejercicio de su sexualidad lesionando el proceso de psicoformación al que por razón de su edad aún se encuentra en proceso de construcción.

\section{Guías teóricas para abordar el problema}

Este trabajo se centra como primer eje teórico a partir de la perspectiva de los derechos humanos considerando que se trata de aquellos cuyo título radica en la personalidad del sujeto o en lo que Dena Romero señala son "derechos suprapositivos y anteriores al estado como órgano creador del ordenamiento jurídico positivo" (2015, p. 46). Esta perspectiva es pertinente para este trabajo de investigación porque establece que los principios a favor de la niñez y la adolescencia deben estar presentes en todos los instrumentos diseñados para proteger los derechos humanos de este grupo poblacional y enfatiza que siendo menores de edad son sujetos de derechos porque pueden solicitar la satisfacción de sus necesidades, se reafirma la corresponsabilidad en cuanto a la atención y crecimiento en un ambiente familiar óptimo y un entorno social de bienestar, por lo que el Estado y la sociedad deben proveer de los recursos necesarios para el cumplimiento de dicho esquema a favor de los menores. Lo anterior es útil en esta investigación para orientar lo relativo al nivel socioeconómico de las víctimas, así como al entorno familiar en el que se desarrollan, ambas situaciones son contempladas como variables independientes dentro del grupo de estudio sobre víctimas.

La trata de personas es un problema multicausal y complejo en el que la literatura señala a la globalización como una cuestión que ha trascendido el espacio económico internacional y ha llegado hasta los aspectos culturales y sociales, generando esquemas de desigualdad que a su vez provocan marcadas desventajas sociales. Así lo señala Della (2014):

El proceso globalizador afecta de muchas maneras varias aristas de nuestra realidad, como lo es en las redes de comunicación, relaciones sociales, comerciales y estatales; la globalización ha afectado el devenir mundial, por lo que ésta no es ya una expresión de economía mundial, sino que ha trascendido a los aspectos de influencia social y que por tanto, las organizaciones del crimen organizado y sus actividades delictivas no han estado exentas a las consecuencias que ha generado este fenómeno (p. 46). 
El crecimiento de la trata de personas como un fenómeno global ha alcanzado tales dimensiones que ahora se considera una forma de esclavitud moderna que se ha actualizado con la implementación de estrategias novedosas para que esta actividad turbia, oscura e ilegal permanezca (Orozco, 2011). Muchos de estos indicios son objeto de análisis en diversos estudios, los cuales señalan como factores preponderantes para la presencia de la trata de personas en México: la dinámica migratoria, la desigualdad social y económica, al igual que la corrupción e impunidad y otros aspectos que generan vulnerabilidad en ciertos grupos demográficos (UNODC, 2014). Asimismo, otros estudios se enfocan en la ubicación geográfica, el nivel socioeconómico, la educación, el entorno de violencia familiar, entre otros factores más que contribuyen a crear un ambiente ameno al florecimiento de este tipo de actividades. Parte de este estudio se enfoca en cristalizar para el lector algunos de los efectos causales de estos elementos a menudo explorados en la literatura sin comprobación fehaciente de su incidencia en el problema de manera específica.

Otro de esos factores es sin duda la tecnología. El Internet juega un importante papel como facilitador para la explotación sexual porque favorece la comercialización de pornografía y su negociación y constituye un espacio casi anónimo para la captación de nuevas víctimas. Como lo establece Orozco cuando expresa que un video de pornografía infantil, por ejemplo, "llega a tener un costo mínimo de 69 dólares por depósito bancario" una vez negociado en el Internet (2011, p. 31). Así pues, la tecnología debe sumarse a las consideraciones que aumentan la magnitud del problema. También se refiere como un factor relacionado con la trata de personas a la migración. La combinación entre migración y trata con fines sexuales es una condición que se presenta en las regiones fronterizas de México, como es en la frontera sur del Estado de Chiapas, donde según Casillas (2011), la actividad del tráfico de personas se despliega en la opacidad pero sirve de proveedor de insumos para el comercio sexual de los migrantes, sobre todo de mujeres, niños, niñas y adolescentes. Son dos actividades que funcionan vinculadas entre sí, pero que obedecen a características distintas porque se trata de fenómenos diferentes. En el extremo norte del Estado de Chihuahua se cuenta con municipios colindantes con el Estado de Nuevo México y Texas de los Estados Unidos de Norteamérica, situación que se ve afectada por el paso de los migrantes que intentan cruzar y que permite inferir que la trata se manifiesta en esta región fortalecida por el factor migratorio.

Otra de las posibles causas en la presencia de trata de personas con fines de explotación sexual en mujeres menores de edad se ubica en lo relativo al entorno familiar. Esto debe ser considerado al abordar el problema en virtud de que las mujeres adolescentes se encuentran en una etapa de desarrollo psicobiológico en proceso de maduración y esto las sitúa en vulnerabilidad (Cruz, 2013). Lo anterior es útil en esta investigación para orientar lo relativo al entorno familiar en el que 
se desarrollan las adolescentes y su nivel socioeconómico. Ambas situaciones se contemplan como variables independientes dentro del estudio sobre variables de incidencia para la vulnerabilidad de las víctimas. Gómez y Ontiveros (2004), por ejemplo, aseveran que,

En el caso mexicano dicha protección a los derechos de niños, niñas y adolescentes, así como para quienes no tienen capacidad para comprender el significado del hecho, debe ser el eje rector de la política criminal, porque ésta representa el esquema de guarda y cuidado de un segmento de la población que representa el futuro de México y que su proceso formativo se realiza con la interacción de los adultos a quienes están encargados así como del Estado, que deberá proveer mediante las instituciones, las bases estructurales requeridas y necesarias para lograr la adecuada satisfacción de las necesidades que comprenden tanto los aspectos de educación y cultura como de salud, seguridad, esparcimiento o vivienda (p. 16).

Es decir, el entorno familiar y afectivo de los menores de edad combina un escenario de corresponsabilidad entre el Estado y la familia, integrándose para proporcionar una base que como fin último logre la adecuada satisfacción de las necesidades de este grupo poblacional, por lo que se vuelve importante considerar estas variables del entorno familiar, en la búsqueda de las causas sobre la prevalencia del fenómeno de la trata de personas con fines de explotación sexual en mujeres adolescentes del Estado de Chihuahua.

De acuerdo con las consideraciones planteadas en los diversos estudios aludidos es que este trabajo se focaliza en tomar como variables centrales cuatro aspectos que circundan los factores en relación con los cuales se explora ubicar la vulnerabilidad de las mujeres víctimas menores de edad, siendo el nivel socioeconómico de las víctimas, su entorno familiar, el papel de las redes sociales y el estatus migratorio, los factores seleccionados para la búsqueda por causas de vulnerabilidad de la trata con fines de explotación sexual en adolescentes del Estado de Chihuahua.

\section{Sección metodológica}

La presente es una investigación que se ubica dentro del paradigma cualitativo que se desarrolla y construye partiendo de la exploración y acercamiento de 28 casos a profundidad para estudiar el fenómeno de la trata con un acercamiento de tipo inductivo. Es decir, el acercamiento metodológico es cualitativo en que la selección es de casos que dan representatividad tipológica al fenómeno y no se basa en la estadística inferencial (Fernández, 2006). Esta es pues una investigación exploratoria de tipo inductivo, como se mencionó anteriormente, porque pretende "obtener un conjunto de proposiciones contrastables que logren representar una 
teoría" (Fernández, 2006, p. 3), que permita encontrar patrones y pasar a la construcción de teorías dinámicas a partir de la recolección de datos, es decir, se parte de lo empírico a lo teórico para lograr la comprensión social de este fenómeno.

Es así que este estudio considera como variables centrales de este el nivel socioeconómico de las víctimas, lo cual se dirige a la hipótesis cuya premisa $\mathrm{H} 1_{\mathrm{v}}$ cuanto más bajo es el nivel socioeconómico de las adolescentes, mayor es la susceptibilidad de caer víctimas de trata de personas con fines de explotación sexual. Como segunda variable y su incidencia en la vulnerabilidad es sobre el entorno familiar de la víctima bajo la hipótesis propuesta como $\mathrm{H} 2$, a mayor carencia afectiva en el entorno familiar se aumenta el riesgo de ser reclutadas por los tratantes de personas.

Seguida de la variable que incluye el rol que juega el uso de las redes sociales bajo la hipótesis $\mathrm{H} 3$, cuanto mayor es el uso de redes sociales, mayor es la situación de vulnerabilidad para que las mujeres de 12 a 18 años sean captadas o enganchadas por los tratantes de personas, con fines de explotación sexual. Todas esas variables son planteadas como independientes. Finalmente se incluye una variable interviniente por ser contextual sobre el estatus migratorio de las víctimas para ver si esta fortalece la presencia de la trata sexual en el Estado de Chihuahua y particularmente en Juárez, bajo la hipótesis auxiliar $\mathrm{H} 1$ a propuesta de que la migración es un factor de incidencia para la trata de personas con fines de explotación sexual en mujeres adolescentes de 12 a18 años. Todas estas son variables centrales aunque no únicas, pero sí las que aquí se consideran como prioritarias para detectar las causas por las que la trata de personas con fines sexuales se manifiesta en las adolescentes de Chihuahua y particularmente de Ciudad Juárez.

Este es, pues, un trabajo de investigación que se diseña con el objetivo de establecer inferencias de carácter descriptivo sobre el fenómeno social de la trata de personas adolescentes porque procura describir cuidadosamente este problema social mediante la acumulación de hechos en forma sistematizada, utilizando observaciones de la realidad para estar en aptitud de obtener otros datos no observados. Dicho de otra manera, se trata de una investigación que pretende especificar las propiedades o características de las víctimas para determinar las circunstancias bajo las que se da el fenómeno de la victimización.

\section{Técnicas de recolección de datos}

En este estudio se eligen como técnicas metodológicas pertinentes las entrevistas semiestructuradas aplicadas a dos víctimas directas de trata con fines sexuales y 24 entrevistas a servidores públicos e integrantes de la sociedad civil avocados en atender este problema, también resulta adecuado utilizar la técnica del análisis 
documental respecto de textos jurídicos como testimoniales de una víctima directa que sobrevivió a la situación y de un testigo en hechos de trata, el seguimiento hemerográfico de 36 notas de prensa digital que proporcionaron datos que sirven de insumos para el estudio y de cuatro videograbaciones en formato DVD sobre causas penales o juicios orales integrados por el delito de trata de personas con fines de explotación sexual. En la siguiente sección se muestran los hallazgos obtenidos y que se basaron en las hipótesis y variables propuestas para este trabajo de investigación, por lo que a continuación se presentan en un apartado que integra las variables utilizadas y su operacionalización, que permitieron escudriñar las causas por las que el lastre social de la trata con fines de explotación sexual se manifiestan en el Estado de Chihuahua.

\section{Factores causales del problema}

Lo que en esta sección se contempla como aspectos o factores contextuales se refiere específicamente a aquellas circunstancias o entorno bajo el cual las mujeres pueden ubicarse en riesgo o vulnerabilidad de trata sexual, explorando las hipótesis que fueron previstas para este trabajo. Entre estos factores se considera el nivel socioeconómico, el entorno familiar de las víctimas, el papel de las redes sociales y la migración como situación que incentiva o fortalece la vulnerabilidad ante la trata. Enseguida se discuten los hallazgos en cada uno de estos factores.

\section{El nivel socioeconómico}

Esta variable se delineó de acuerdo con algunos estudios previos que coinciden en que cambios económicos han tenido gran impacto generando desigualdad social y acarreando desventajas de tipo económico en el que las mujeres, niñas y adolescentes se ven mayormente expuestas (Acharya y Salas, 2005); por lo tanto, aquí se pretende establecer si el nivel socioeconómico impacta como aspecto que genera vulnerabilidad ante la trata y es por lo que en esta sección se delinea la búsqueda sobre los 29 casos a los que se tuvo acceso respecto del nivel socioeconómico de las víctimas, enmarcando la indagación bajo la variable que se operacionaliza mediante la ubicación de la vivienda y el nivel de escolaridad de las víctimas. Se expone aquí que de los 29 casos que integran el estudio, solo uno es considerado como nivel medio y en 28 de estos se encuentra que su nivel socioeconómico es bajo debido a que el lugar de la vivienda de las víctimas corresponde a zonas referidas en situación de pobreza como en las colonias Guadalajara Izquierda, Revolución Mexicana, Fronteriza, Anapra y en los dos casos de la ciudad de Chihuahua los hallazgos muestran que se trata de víctimas en situación de calle y que fueron llevadas a un albergue. 
El acercamiento que se tuvo con una de las víctimas revela que su vivienda se encuentra localizada en la colonia La Chaveña en la zona centro de Ciudad Juárez. En el caso de la segunda víctima entrevistada, su vivienda se localizaba en la calle Cristóbal Colón, también en la zona centro y en relación con la víctima 3 de la que se obtuvo información en la lectura de su testimonio judicial, ella misma especifica haber vivido en los hoteles del centro de Ciudad Juárez desde los doce años porque no tenía dinero para quedarse en algún otro lugar. En los dos casos referidos por la Fiscalía especializada en delitos cometidos por razones de género, las víctimas vivían en la prolongación de la Avenida 16 de septiembre, que también corresponde a la zona del poniente de Ciudad Juárez, una zona con altos niveles de pobreza. Además de que el ministerio público que proporcionó la información de estos casos menciona específicamente la descripción de la vivienda de las adolescentes:

...nos damos cuenta que la situación en las que ellas viven son muy precarias, mencionan que vivían en cuartitos de tierra incluso con piso de tierra, la de 17 años si en una casa más grande pero pues... ahí vivan sus hermanos, con sus hijos y esa casa es la que les habían dejado sus papás... (comunicación personal, 9 de enero de 2017)².

En el mismo sentido la Comisión Nacional para prevenir y erradicar la violencia contra las mujeres (CONAVIM) en Ciudad Juárez, tiene identificado que en los casos de trata, las víctimas son mujeres que pertenecen a un sector poblacional vulnerado económicamente, que a veces no pueden estudiar y que buscan ingresar al campo laboral desde edades muy tempranas, ya que abandonan la escuela llegando a cursar solamente la primaria y en otros casos ingresan a secundaria sin terminarla (comunicación personal, Zulay Abud, 12 de octubre de 2016) 3 $^{3}$ El señalamiento anterior es repetido por los demás integrantes de la comunidad gubernamental y de organismos de la sociedad civil que fueron entrevistados y que coinciden en expresar que su experiencia les indica que tratándose de víctimas de trata, todas corresponden a un nivel socioeconómico bajo. Así se expone el resultado sobre esta variable en la tabla 1.0.

2 El agente del ministerio público que proporciona esta información solicitó expresamente no ser identificado con su nombre. Así lo dejó asentado en la carta de consentimiento informado que se le entregó previo a la entrevista.

3 Información proporcionada por la Licda. Zulay Abud Esparza, Directora General adjunta de Representaciones temporales de la Comisión para prevenir y erradicar la violencia contra las mujeres, órgano desconcentrado de la Secretaría de Gobernación. 
Tabla 1.0

Nivel socioeconómico de las víctimas

\begin{tabular}{|c|c|c|c|}
\hline $\begin{array}{l}\text { Fuente de } \\
\text { información }\end{array}$ & $\begin{array}{l}\text { Número } \\
\text { de casos }\end{array}$ & Vivienda & Escolaridad \\
\hline Arroyo El Navajo & 11 & $\begin{array}{l}\text { Viviendas ubicadas en } \\
\text { colonias Guadalajara } \\
\text { Izquierda, Fronteriza, } \\
\text { Revolución Mexicana, } \\
\text { Anapra }\end{array}$ & $\begin{array}{c}\text { Algunas llegaron hasta } \\
\text { secundaria }\end{array}$ \\
\hline \multirow{5}{*}{$\begin{array}{l}\text { Subprocuraduría de protección } \\
\text { a N, N y A, distrito Bravos }\end{array}$} & 1 & Bajo & Sin asistir a la escuela \\
\hline & 1 & Bajo & Sin asistir a la escuela \\
\hline & 1 & Bajo & Sin asistir a la escuela \\
\hline & 1 & $\begin{array}{c}\text { Bajo, colonia Independencia } \\
\text { II }\end{array}$ & Secundaria \\
\hline & 1 & Bajo, Senderos de San Isidro & Secundaria \\
\hline $\begin{array}{l}\text { Procuraduría de protección a N, } \\
\text { N y A del Estado de Chihuahua }\end{array}$ & 1 & $\begin{array}{l}\text { Bajo, hoteles de la zona } \\
\text { centro, sin vivienda }\end{array}$ & Sin asistir a la escuela \\
\hline \multirow{2}{*}{$\begin{array}{c}\text { Fiscalía de atención a víctimas } \\
\text { y ofendidos del delito }\end{array}$} & 1 & Nivel bajo & Primaria \\
\hline & 1 & $\begin{array}{l}\text { Nivel medio, Colonia } \\
\text { División del Norte }\end{array}$ & Secundaria \\
\hline Víctima 1 & 1 & $\begin{array}{l}\text { Bajo, colonia La Chaveña, } \\
\text { zona centro de la ciudad }\end{array}$ & Secundaria \\
\hline \multirow{4}{*}{ Víctima 2} & 1 & \multirow{4}{*}{$\begin{array}{c}\text { Sin vivienda, habitan en los } \\
\text { hoteles del centro }\end{array}$} & \multirow{4}{*}{ Sin asistir a la escuela } \\
\hline & 1 & & \\
\hline & 1 & & \\
\hline & 1 & & \\
\hline Víctima 3 & 1 & $\begin{array}{c}\text { Sin vivienda, habitan en los } \\
\text { hoteles del centro }\end{array}$ & No fue a la escuela \\
\hline \multirow{2}{*}{ Libres por amor A.C. } & 1 & En situación de calle & \multirow{2}{*}{ Sin asistir a la escuela } \\
\hline & 1 & En situación de calle & \\
\hline \multirow{2}{*}{$\begin{array}{c}\text { Fiscalía de delitos cometidos } \\
\text { contra la mujer por razones de } \\
\text { género }\end{array}$} & 1 & $\begin{array}{c}\text { Prol. Av. } 16 \text { de septiembre, } \\
\text { zona centro }\end{array}$ & \multirow{2}{*}{ Primaria } \\
\hline & 1 & $\begin{array}{c}\text { Prol. Av. } 16 \text { de septiembre, } \\
\text { zona centro }\end{array}$ & \\
\hline
\end{tabular}

Fuente: elaboración propia. 
Lo expuesto indica que la variable relativa al nivel socioeconómico se operacionalizó con las características de ubicación de la vivienda, como se especificó, pero también por el nivel de escolaridad de las víctimas, encontrándose que en este último el resultado indica que el nivel de escolaridad no excede en ninguno de los casos de la escuela secundaria. Lo expuesto se integra en una consolidación de los datos que se presentan en la tabla 1.0, donde se muestran hallazgos sobre veintiséis de los veintiocho casos, ya que en dos de estos no se tuvo acceso a esa información porque solamente se informó que se trató de dos menores de edad sujetos a pornografía infantil (comunicación personal, 4 de enero de 2017) , $^{4}$ sin poder ampliar la información en razón de que es derivada de un expediente jurídico con proceso judicial abierto en el momento de realizar la entrevista. En cuanto a los casos obtenidos de la revisión de expedientes integrados por la Subprocuraduría de protección auxiliar de niñas, niños y adolescentes para el distrito judicial Bravos, correspondientes a cinco casos en los que la valoración que determina el nivel socioeconómico bajo es derivada del trabajo realizado por personal de dicha dependencia y en algunos casos de las fotografías tomadas a la vivienda de la víctima y que obran en las actuaciones del personal de trabajo social adscrito a dicha agencia gubernamental ${ }^{5}$.

De la lectura de los datos se deriva que la primera hipótesis $\mathrm{H} 1_{\mathrm{v}}$ cuya premisa es que cuanto más bajo es el nivel socioeconómico de las adolescentes, mayor es la susceptibilidad de caer víctimas de trata de personas con fines de explotación sexual, se fortalece, toda vez que durante el levantamiento del trabajo de campo, de los actores entrevistados y de quienes tienen contacto directo con esta problemática, solamente la Fiscalía de Atención a víctimas y ofendidos del delito menciona un solo caso con un menor sujeto a trata sexual cuyo nivel socioeconómico es considerado como medio. Sin embargo, cabe mencionar que la hipótesis planteada no excluye que la trata se presente en víctimas potenciales de un nivel socioeconómico medio o alto, pero los hallazgos de este estudio indican que el fenómeno se actualiza preponderantemente con adolescentes de nivel socioeconómico bajo, situación enlazada a la necesidad de abandonar la escuela y buscar empleo.

4 El servidor público de Procuraduría General de la República solicitó no ser identificado, concedió la entrevista el día 4 de enero de 2017 en domicilio particular distinto al de las instalaciones de la dependencia.

5 Cabe mencionar que en los casos cuya fuente de información es a partir del acercamiento con la Subprocuraduría de atención a niños, niñas y adolescentes del distrito Bravos, la información fue obtenida en tres fases, la primera en la que la encargada de la unidad pide que se solicite por escrito y la respuesta se obtiene de la misma forma estableciendo que de los tres casos documentados en esa oficina todos corresponden a nivel socioeconómico bajo sin detallar características de vivienda; lo anterior es así ya que el titular de dicha dependencia solicitó que fuera de esa manera y no en entrevista de viva voz. Posteriormente se admite la revisión física de los expedientes en dos sesiones posteriores, lo que permitió ahondar en los tres casos ya mencionados y agregar otros más permitiéndose a quien investiga tomar notas, pero con la advertencia de no poder identificar el número de expediente ni usar los nombres de los involucrados. 
El hecho de que todos los actores entrevistados para esta investigación y que se encuentran avocados en la atención, prevención, sanción y erradicación de la trata en el Estado de Chihuahua, no tengan documentados más casos de personas sujetas a explotación sexual por trata de un nivel socioeconómico medio o alto puede fundamentarse en el argumento que Norma Ledezma ${ }^{6}$ señala al expresar:

El bajo nivel socioeconómico sí es un común denominador, o sea no quiere decir que una persona muy rica no pueda eh... este... no pueda ser víctima de esto. Sin embargo, si fuera muy rica económicamente, si una persona de un alto estrato social no lo reportaría, porque le hablan a nivel Gobernador y ponen helicópteros y saben los malandros que van a ser detenidos...

...a mí me llega la gente, obviamente la gente del barrio, llegan en camión, la gente que... gente donde vivo yo, mi barrio, mi gente o sea nosotros apoyamos al sector más vulnerable de la sociedad, el que no tiene unas oportunidades de ser atendidos a niveles superiores, donde el nombre de su hija, su apellido eh... no es reconocido. Es cualquier apellido. Este... los de apellido de alcurnia pues ellos tienen otros tratos y otros abogados y otras maneras de resolverlo, en caso de que así fuera. Sin embargo yo, ni han llegado ni conozco casos de trata, habrá secuestros. Porque son otro delito que es más propio para ellos, pero no de trata. O sea los delincuentes no se van a meter con la hija de un funcionario público, no, o sea igual no andan en camiones de noche, no trabajan en maquiladoras, ni van a fiestas en el centro de la ciudad, ni al mercado a conseguir trabajo o a este... conseguir ropa. Ni a comer a los puestos de ahí... por ahí. Esa gente no va ahí, entonces pues ellos no serían víctimas de eso (comunicación personal, Norma Ledezma, 15 de enero de 2017).

La evidencia recogida y las entrevistas indican que los casos de trata sexual en un nivel socioeconómico medio o alto se atienden de otra manera, sin pasar por los estándares de atención institucional y que, por lo mismo, no logran ser documentados ni reflejados en los datos estadísticos del problema. Es así como se puede puntualizar que la hipótesis relacionada con el estatus socioeconómico tiene una fuerza explicativa importante al establecerse como causa de vulnerabilidad ante la trata el nivel socioeconómico de las adolescentes. Ahora es necesario revisar el comportamiento de la segunda variable respecto de la segunda hipótesis planteada para esta investigación y que se refiere al entorno familiar.

6 Norma Ledezma es presidenta de la organización civil sin fines de lucro llamada Justicia para nuestras hijas, que tiene su radio de acción en todo el Estado de Chihuahua realizando acciones de acompañamiento con víctimas directas e indirectas. 


\section{El entorno familiar de las víctimas}

Es importante considerar que uno de los parámetros establecidos para este estudio se concentra en las edades de las víctimas de trata sexual en Chihuahua, razón por la que se delimitó la investigación respecto del corte etario sobre los 12 a 18 años de edad que abarca la edad adolescente y también porque corresponde a uno de los ejes teóricos sobre derechos humanos desde la perspectiva de la doctrina de la protección integral de niños, niñas y adolescentes donde expresamente contempla que el Estado, la familia y la sociedad deben articular acciones, políticas y programas para garantizar que este grupo poblacional no se vea expuesto ni sujeto a condiciones que transgredan sus derechos humanos (Gómez Tagle y Ontiveros, 2004).

Con el sustento teórico de los Derechos Humanos y la teoría sustantiva de la Doctrina de la protección integral, es como se delinea la variable relativa al entorno familiar de las víctimas, la cual se operacionaliza sobre parámetros concretos de comunicación con los padres, el apoyo que estos dan a sus hijos menores, y si las víctimas viven en un entorno de hostilidad y rechazo. Esto es para visualizar si al existir un ambiente familiar con características como las mencionadas se genera vulnerabilidad de trata sexual o si tales condiciones, que se alejan de lo previsto por esta teoría sustantiva, quedan excluidas como aspecto que crea vulnerabilidad individual.

Una medida precisa es imposible de lograr, pero es posible determinar un patrón sobre el estado del entorno familiar de las víctimas y su victimización. En esta sección se busca conocer si desde el ambiente familiar se establecen aspectos o causales que incidan en la vulnerabilidad individual de las víctimas para ser captadas por los tratantes, por lo que la variable del entorno familiar adverso logra operacionalizarse a partir de si las víctimas tenían comunicación con sus padres y si en su ambiente inmediato hay rasgos de hostilidad y rechazo. Los parámetros con los que se mide el entorno familiar se muestran en la tabla 2.0.

Tabla 2.0

Entorno familiar de las víctimas

\begin{tabular}{|c|c|c|c|}
\hline $\begin{array}{c}\text { Fuente de } \\
\text { información }\end{array}$ & $\begin{array}{c}\text { Número } \\
\text { de Casos }\end{array}$ & $\begin{array}{c}\text { Comunicación } \\
\text { con padres }\end{array}$ & $\begin{array}{c}\text { Ambiente de } \\
\text { hostilidad y } \\
\text { rechazo }\end{array}$ \\
\hline \multirow{2}{*}{ Arroyo El Navajo } & 1 & No & Sí \\
\cline { 2 - 4 } & 1 & Sí & No \\
\cline { 2 - 4 } & 9 & Sin datos & Sin datos \\
\hline
\end{tabular}

150 (29) $\begin{aligned} & \text { Revista Latinoamericana de Derechos Humanos } \\ & \text { Volumen 29 (1), I Semestre } 2018 \text { (ISSN: 1659-4304) } \\ & \text { (EISSN: 2215-4221) }\end{aligned}$ 


\begin{tabular}{|c|c|c|c|}
\hline $\begin{array}{l}\text { Fuente de } \\
\text { información }\end{array}$ & $\begin{array}{l}\text { Número } \\
\text { de Casos }\end{array}$ & $\begin{array}{c}\text { Comunicación } \\
\text { con padres }\end{array}$ & $\begin{array}{l}\text { Ambiente de } \\
\text { hostilidad y } \\
\text { rechazo }\end{array}$ \\
\hline \multirow{5}{*}{$\begin{array}{c}\text { Subprocuraduría de protección a niñas, } \\
\text { niños y adolescentes, distrito judicial } \\
\text { Bravos }\end{array}$} & 1 & $\mathrm{No}$ & Sí \\
\hline & 1 & $\mathrm{No}$ & Sí \\
\hline & 1 & No & Sí \\
\hline & 1 & $\mathrm{No}$ & Sí \\
\hline & 1 & $\mathrm{No}$ & Sí \\
\hline $\begin{array}{c}\text { Procuraduría de protección a N, N y A de } \\
\text { Chihuahua }\end{array}$ & 1 & $\mathrm{No}$ & Sí \\
\hline \multirow{2}{*}{$\begin{array}{l}\text { Fiscalía de atención a víctimas } \\
\text { y ofendidos del delito }\end{array}$} & 1 & No & Sí \\
\hline & 1 & Sí & $\mathrm{No}$ \\
\hline Víctima 1 & 1 & No & Sí \\
\hline \multirow{4}{*}{ Víctima 2} & 1 & No & Sí \\
\hline & 1 & $\mathrm{No}$ & Sí \\
\hline & 1 & $\mathrm{No}$ & Sí \\
\hline & 1 & $\mathrm{No}$ & Sí \\
\hline Víctima 3 & 1 & No & Sí \\
\hline \multirow{2}{*}{ Libres por amor A.C. } & 1 & No & Sí \\
\hline & 1 & $\mathrm{No}$ & Sí \\
\hline \multirow{2}{*}{$\begin{array}{l}\text { Fiscalía de delitos cometidos contra } \\
\text { la mujer por razones de género }\end{array}$} & 1 & Sí & Sí \\
\hline & 1 & $\mathrm{No}$ & Sí \\
\hline
\end{tabular}

Fuente: elaboración propia. El valor No indica sin comunicación con padres o no vivir en ambiente de hostilidad y rechazo. El valor Sí indica comunicación con padres y vivir en ambiente de hostilidad y rechazo.

Referente a los cinco casos informados por la Subprocuraduría de protección de los derechos de niños, niñas y adolescentes del distrito judicial Bravos, el resultado marca que se trata de cinco víctimas femeninas, dos de ellas en situación de calle y, por lo tanto, fuera de un esquema de comunicación con padres y sin redes de apoyo, lo que refleja un ambiente de hostilidad y rechazo que las orilló a abandonar el hogar y esto motiva su situación de calle, mientras que la tercera víctima fue agredida sexualmente por su padre y vivió con sus abuelos y después con su madre y padrastro, lo que indica un ambiente de hostilidad y rechazo; el cuarto caso indica en la comparecencia de la víctima no tener mamá porque falleció y a pesar de tener a su padre externa que frecuentemente se queda en casa de su hermano, donde fue encauzada a la prostitución por parte de la suegra de este; el último caso reportado por esta agencia gubernamental es de una víctima con un entorno familiar adverso debido a que nunca ha visto juntos a sus padres, 
no estudia y su madre es muy estricta y a pesar de que sabe que se prostituye no le brinda la atención debida y no quiere vivir con su madre porque las peleas están presentes y expresa haber usado marihuana, cocaína, agua celeste y piedra desde los catorce años (Revisión de expedientes, 7 de septiembre de 2017). Todo esto es indicativo de un ambiente de rechazo por parte de sus familias.

La Procuraduría de protección a los derechos de niñas, niños y adolescentes del Estado de Chihuahua menciona un caso que corresponde a una mujer adolescente de quince años, que vive en los hoteles del centro y pertenece a una banda del crimen organizado que opera en la zona centro de Ciudad Juárez. Se trata de una menor que ha estado bajo situación de trata sexual, pero que a su vez se encarga de "administrar un picadero" del centro de la ciudad; en este caso no hay registro de relación de ningún tipo con sus padres, no tiene hogar ni domicilio establecidos, ha vivido en el ambiente delincuencial en el que el vínculo con consumo y distribución de drogas está presente y ella misma no se asume como víctima a pesar de estar bajo prostitución forzada. La descripción de la situación anterior conlleva a establecer que se trata de una adolescente con carencias en su entorno familiar anterior, que no tiene una relación afectiva con sus padres y que ha vivido un ambiente de hostilidad y rechazo. Ella prefiere vivir como parte de la red criminal que además la utiliza para enganchar a otras adolescentes y luego someterlas a la trata sexual (comunicación personal, Lic. César Juárez, 25 de mayo de 2017) ${ }^{8}$.

Se incluyen también los dos casos referidos por la Fiscalía de atención a víctimas y ofendidos del delito, los cuales corresponden a dos menores de edad varones. El primero de ellos es alguien que fue rechazado por su familia en razón de su homosexualidad y que, por lo tanto, él mismo acepta ser expuesto a prostituirse como forma de subsistir. El entorno familiar de este menor es adverso y él mismo señaló en su comparecencia que su mamá llegó a consentir en prostituirlo con un señor por $\$ 550.00$ y expresa haber sido violado cuando tenía 5 años. El segundo caso derivado de esta fuente de información y que se complementa con la revisión de expedientes en la Subprocuraduría de protección de los derechos de niños, niñas y adolescentes del distrito judicial Bravos, corresponde también a un varón menor de edad, pero que sí tiene un entorno familiar de cuidado y protección, fue captado de forma violenta y expuesto a explotación sexual. Su familia reportó su

7 Picadero es el término utilizado para referirse al inmueble donde se venden y se consumen drogas.

8 La entrevista fue proporcionada por el titular de la Procuraduría de protección a los derechos de niñas, niños y adolescentes del Estado de Chihuahua, que depende del sistema DIF (Desarrollo Integral de la Familia) a nivel estatal. 
desaparición y estuvo pendiente del proceso de búsqueda y localización (comunicación personal, 9 de noviembre de 2016) ${ }^{9}$.

En el caso de una de las víctimas entrevistadas para este estudio, ella misma expresa que no tiene padre y la relación con su madre era muy mala, pues había golpes y regaños sistemáticamente. Su hermana se dedicaba a la prostitución y todo ello constituye un factor de expulsión para salirse de su casa (comunicación personal, 27 de octubre de 2016) ${ }^{10}$. La segunda víctima entrevistada refiere cuatro casos de adolescentes menores y sujetas a explotación sexual en los hoteles del centro de Ciudad Juárez, pero declara que tres de ellas fueron llevadas ahí por sus madres, que son mujeres dedicadas al sexo servicio, con problema de adicción a las drogas, y que llevan a sus hijas ahí para que consigan dinero y con eso sostener su consumo de drogas. La última referencia es sobre una menor que vivía también en los hoteles Verde y Amarillo, sin entorno familiar, viviendo en el ambiente delincuencial entre la prostitución forzada y el consumo de drogas (comunicación personal, 2 de marzo de 2017) ${ }^{11}$. De igual manera, tratándose de otra víctima, los hallazgos orientan que se diagnostica dentro de un entorno familiar adverso debido a que no tiene relación afectiva con sus padres o con algún familiar que le brinde apoyo, incluso para la atención médica que requiere porque padece de epilepsia, quedando expuesta a ser captada para prostitución forzada y la venta de drogas por tratantes de la zona centro de Ciudad Juárez (declaración testimonial en juicio oral 267/2014).

La problemática de la trata sexual no se concentra únicamente en el municipio de Juárez, es un fenómeno presente en otras zonas del Estado de Chihuahua, tales como en la sierra o en municipios referidos de Parral, Delicias y la ciudad de Chihuahua capital, donde la psicóloga de la agrupación Libres por amor A.C. informa específicamente de una niña de 10 años y un niño de 7 años, hermanos, y a los que su madre consentía en entregarlos para la explotación sexual a cambio de dinero. Se asevera también que vivían en un ambiente de violencia, porque

9 El servidor público entrevistado como representante de la Fiscalía de atención a víctimas y ofendidos del delito, al firmar el consentimiento informado expresamente manifestó no querer ser identificado por nombre ni por el cargo que desempeña en dicha agencia de gobierno, lo que se respeta en la narrativa de este trabajo y solamente se menciona la fecha de la entrevista que se realizó en las instalaciones de la misma agencia gubernamental ubicada en Eje Vial Juan Gabriel y calle Aserraderos de Ciudad Juárez.

10 En apego a las consideraciones éticas de la investigación social se omite el nombre de la víctima. La entrevista fue concedida en el albergue de la organización Sin Violencia A.C. que utiliza como refugio al que fue canalizada por la Fiscalía General del Estado. Estuvieron presentes la directora del albergue y la abogada de este.

11 La víctima que aquí se identifica como 2 fue contactada por medio de una congregación religiosa enfocada en ayudar a mujeres en prostitución en Ciudad Juárez. También se omite el nombre de la entrevistada por las consideraciones éticas del estudio. La entrevista se realizó en la casa que tienen las Hermanas Oblatas del Santísimo Redentor ubicada en la Avenida Vicente Guerrero esquina con calle Paraguay de Ciudad Juárez. 
refieren que su madre los golpeaba y obligaba a estar con señores para poder darles de comer a todos sus hermanitos y además también se dedicaba al sexo servicio (comunicación personal, Marisol Enríquez, 8 de diciembre de 2016), lo que expone potencialmente un entorno familiar adverso para estos menores. Es interesante observar cómo estos entornos se cruzan también con la propia carencia económica, suponiendo que estas dos variables interactúan entre sí de manera compleja, exacerbando su efecto sobre la vulnerabilidad.

Hay otros dos casos más informados por un colaborador adscrito a la Fiscalía de atención de delitos cometidos contra la mujer por razones de género (FEM), los cuales corresponden a dos mujeres adolescentes y amigas. Una de ellas sí presenta indicios de comunicación con su madre, pero no tiene padre. Vive con su madre y dos hermanitos menores y aunque sí tiene comunicación con ella su situación es complicada porque no puede estudiar por tener que cuidar a sus hermanos. La familia vive en condiciones muy precarias económicamente y todo ello la sitúa en un ambiente hostil, a pesar de mostrar una relación afectiva con su madre. Su visión hacia el futuro se centra en obtener bienes materiales como un celular o ropa a los que no tiene acceso por no tener posibilidades económicas para ello. La segunda víctima vive en la casa que dejaron sus padres pero con un hermano mayor, alcohólico que la golpea y maltrata (comunicación personal, 9 de febrero de 2017) ${ }^{12}$ y esto la ubica como una persona adolescente que se está desarrollando en un ambiente negativo de hostilidad y en ausencia de apoyo que guíen su desarrollo psicosocial.

Del análisis sobre los datos obtenidos en los casos expuestos, se obtiene que en diecisiete de estos hay un evidente entorno familiar adverso, sujeto a ausencia de armonía familiar, relación afectiva con los padres, en medio de un ambiente hostil, mientras que en otros dos casos sí existe o existía una relación de cuidado y apoyo de los padres a sus hijos menores y en 9 de los casos no se pudo tener acceso a este nivel de información, porque se trata de víctimas sin vida y no se cuenta con datos institucionales a este nivel. Por lo tanto, la hipótesis planteada como Hv2 cuya premisa es que a mayor carencia afectiva en el entorno familiar mayor el riesgo de ser reclutadas por los tratantes de personas, muestra fuerza explicativa de acuerdo con los hallazgos mostrados en los casos que integran este estudio.

12 El servidor público entrevistado no autoriza ser identificado por su nombre ni por el cargo que desempeña en la agencia gubernamental a la que está adscrito. La entrevista se realizó en un lugar distinto al de la dependencia. 


\section{El papel de las redes sociales}

Al delinear el marco referencial bajo el que se pensó guiar el estudio del problema que aquí se plantea, se delimitó que en el eje teórico de la globalización es necesario ubicar lo relativo a las tecnologías de la información y en específico sobre el uso de redes sociales. Esta decisión deriva de la lectura de algunos argumentos esgrimidos por diversos estudios en los que se ha encontrado que la actividad de la trata de personas es favorecida por la rapidez con la que actualmente se despliegan los mecanismos de enganche que se sirven de estas herramientas de comunicación para establecer contacto primario con las potenciales víctimas o en su caso realizar la explotación sexual utilizando las diversas posibilidades cibernéticas que existen convirtiéndolas en una arena para la pornografía con menores de edad (Negrete, 2015).

Otros estudios enfocan su análisis en la importancia que este medio de comunicación, sus plataformas y la información contenida en todas ellas como un elemento que abona al problema de la trata en virtud de que se ha vuelto un medio para la comercialización de pornografía y que ayuda a entablar un robusto sistema financiero que obtiene ganancias asociadas a esta actividad (Chávez, 2011). Desde esa perspectiva, se delineó como otra variable independiente que el uso de redes es un factor que genera mayor vulnerabilidad para ser captadas por los tratantes por su afición al uso de redes sociales, que a veces están fuera del entendimiento de sus padres. Por esto se planteó medir esta variable a partir del ingreso y el tipo de uso que les dan a las redes sociales, teniendo como resultado el siguiente consolidado de los casos analizados y que se muestra en la tabla 3.0:

Tabla 3.0

Redes sociales y trata de personas

\begin{tabular}{|c|c|c|c|}
\hline $\begin{array}{c}\text { Fuente de } \\
\text { información }\end{array}$ & $\begin{array}{c}\text { Número } \\
\text { de casos }\end{array}$ & $\begin{array}{c}\text { Uso de redes } \\
\text { sociales }\end{array}$ & Tipo de uso \\
\hline Arroyo El Navajo & 11 & No & No \\
\hline $\begin{array}{c}\text { Subprocuraduría de protección a N, N y } \\
\text { A, distrito judicial Bravos }\end{array}$ & 1 & No & No \\
\cline { 2 - 4 } & 1 & No & No \\
\hline & 1 & No & No \\
\hline & 1 & No & No \\
\hline
\end{tabular}




\begin{tabular}{|c|c|c|c|}
\hline $\begin{array}{c}\text { Fuente de } \\
\text { información }\end{array}$ & $\begin{array}{c}\text { Número } \\
\text { de casos }\end{array}$ & $\begin{array}{c}\text { Uso de redes } \\
\text { sociales }\end{array}$ & Tipo de uso \\
\hline $\begin{array}{c}\text { Procuraduría de protección a N, N y A de } \\
\text { Chihuahua }\end{array}$ & 1 & No & No \\
\hline Víctima 1 & 1 & No & No \\
\hline Víctima 2 & 1 & No & No \\
\cline { 2 - 4 } & 1 & No & No \\
\cline { 2 - 4 } & 1 & No & No \\
\cline { 2 - 4 } & 1 & No & No \\
\hline Víctima 3 & 1 & No & No \\
\hline \multirow{2}{*}{$\begin{array}{c}\text { Libres por amor A.C. } \\
\text { Fiscalía de delitos cometidos contra la } \\
\text { mujer por razones de género }\end{array}$} & 1 & No & No \\
\cline { 2 - 4 } & 1 & So & $\begin{array}{c}\text { Facebook y } \\
\text { WhatsApp }\end{array}$ \\
\hline
\end{tabular}

Fuente: elaboración propia. El valor No significa no intervención de uso de redes sociales y Sí indica el uso de redes sociales.

Los datos presentados indican que solo en dos casos se utilizaron las redes sociales Facebook y WhatsApp y sirvieron de mecanismo de enganche. Hay evidencia de que para la explotación obligaron a las víctimas a tomarse fotos y enviarlas por esos medios e inclusive, sus explotadores les regalaron el teléfono celular que utilizaban para localizarlas y llevarlas con los clientes (comunicación personal, 9 de febrero de 2017). Esto implica que las redes no son solo un elemento de enganche potencial, sino que también sirven para conducir el negocio de la explotación sexual con menor riesgo de ser captados porque se hace a manera de transacción privada por teléfono.

Sin embargo, durante la etapa de trabajo de campo se encontró que existe un auge importante sobre casos de sexting a partir del año 2016 en el que se reportan denuncias penales en la Fiscalía General del Estado, con motivo de jóvenes adolescentes que son contactadas por Facebook y WhatsApp para subir fotografías y enviarlas en grupos cerrados. La Fiscalía señala que hay más de 10 grupos en Facebook centrados en el intercambio de pornografía resaltando la existencia del denominado "femmes rojo" y "femmes fatales" como comunidades cibernéticas donde las adolescentes acceden a ver y participar en pornografía, lo que es 
considerado por los operadores gubernamentales como una actividad previa a la trata sexual, toda vez que el administrador de dichos grupos sube las imágenes y se asume que pueden ser vendidas con posterioridad. Esta es una situación que se investiga actualmente.

En el momento de realizar el levantamiento de trabajo de campo, se encuentra que de enero de 2016 a mayo de 2017 existen poco más de veinte carpetas de investigación por sexting en redes sociales, de las que se tiene referencia de que todas las víctimas son mujeres con edades entre los 15 a 20 años, a excepción de un caso donde la víctima es una mujer adulta de 45 años. La escolaridad de las participantes oscila entre secundaria y preparatoria, pero un hallazgo importante es el del entorno familiar de este tipo de víctimas porque no se refiere como un ambiente adverso en el hogar, sino que más bien se trata de carencia de supervisión por parte de los padres a sus menores hijas (comunicación personal, Adán Herrera, 13 de mayo de 2017) ${ }^{13}$. Esto refleja ausencia de control y desconocimiento del uso que les dan a las redes sociales las jóvenes, aun y cuando se encuentran dentro del hogar y bajo la supuesta supervisión de los padres. En cuanto al nivel socioeconómico se les considera de clase media a clase media alta, de acuerdo con el tipo de escuelas donde estudian y generalmente se encuentran en el seno de una familia con patrones clasemedieros, de lo cual también se infiere que se trata de jóvenes con teléfonos inteligentes y acceso a Internet. Esto implica también que existen dos vías, la que indican las primeras variables, donde la condición socioeconómica y el entorno familiar son importantes, y una segunda vía donde las redes juegan un papel importante.

Por lo tanto, y derivado de lo antes expuesto, la hipótesis $\mathrm{H} 3{ }_{\mathrm{v}}$ cuya premisa es que cuanto mayor es el uso de redes sociales mayor es la situación de vulnerabilidad para que las mujeres de 12 a 18 años sean captadas o enganchadas por los tratantes de personas, se confirma parcialmente porque a pesar de que en la integración de los casos que aquí se analizan, solo resaltan dos situaciones relacionadas directamente con el uso de redes sociales y las características socioeconómicas de las jóvenes participantes son diferentes a las de otros casos. Pero se puede afirmar que el problema de la trata se fortalece o se nutre de etapas previas en las que los grupos cerrados a través de Facebook, WhatsApp, Instagram, Snapchat y otros más sirven como medios preparatorios para consolidar la trata con fines sexuales, y que como se refiere por la comunidad gubernamental, estos medios constituyen una herramienta que fortalece el problema por la rapidez con la que circulan las imágenes o por la facilidad que brindan para entablar contacto inicial y permanente, pero también

13 El entrevistado Adán Herrera es Licenciado en Criminología y está adscrito a la Fiscalía General del Estado de Chihuahua en la Unidad de delitos sexuales y trata de personas. 
furtivo, con las potenciales y actuales víctimas. Los casos canalizados incluso sugieren que esto puede ocurrir aun bajo la supervisión física de los menores y en condiciones familiares sin muchos problemas.

\section{Migración y trata sexual en Chihuahua}

El Estado de Chihuahua es el más grande de la República, con una extensión territorial que representa el $12.62 \%$ del territorio nacional y que según el Instituto Nacional de Estadística y Geografía para el año 2015 cuenta con una distribución poblacional de 1804299 mujeres y 1752275 hombres, y con tres municipios que hacen frontera con Nuevo México y Texas: Puerto Palomas, Juárez y Ojinaga. El INEGI registra que para el 2010 el flujo migratorio de carácter nacional fue de 58 334 personas procedentes de otros estados del país mientras que sobre migración internacional, para ese mismo año, de cada 100 migrantes 93 migran a los Estados Unidos de Norteamérica. De los tres municipios fronterizos, el de Juárez es el más grande de Chihuahua, con una población de 1332131 habitantes que representa el $39.11 \%$ de la población del Estado, lo que lo ubica como el segundo municipio más poblado del norte de México y como la ciudad más importante de Chihuahua ${ }^{14}$.

Tales características geográficas y demográficas conllevan a la necesidad de revisar si el aspecto migratorio en la entidad, particularmente en Ciudad Juárez, incide también en la presencia de la trata sobre los migrantes nacionales que se quedan a residir en el Estado o los que emigran con la intención de llegar a Estados Unidos. Con lo anterior se pretende dimensionar si actualmente el estatus migratorio incide como causal que fortalece la presencia del fenómeno en estudio, tal como se refiere por algunas investigaciones que señalan la presencia de la explotación sexual en Ciudad Juárez propiciada por la repatriación de menores o porque hay adolescentes que cruzan a la ciudad de El Paso, Texas, para prostituirse (Azaola, 2003), o bien, si actualmente dicha condición ha variado perdiendo fuerza vinculatoria con el problema porque disminuyeron los flujos migratorios debido a una "disminución de la capacidad de atracción que había tenido la ciudad donde entre el período del año 2000 y 2010 pasó de un 12 por ciento a un 2.8 por ciento" (Rubio, 2011, p. 9). Estos son los argumentos que se utilizan para revisar si la trata se manifiesta con fuerza vinculatoria del problema debido al estatus migratorio de las víctimas.

De los casos que se han analizado para el presente estudio el hallazgo relevante es el caso de una víctima nacida en Tamaulipas y otro más donde la víctima es originaria de Veracruz pero tienen residencia permanente en la ciudad. En ninguno

14 Información tomada del portal digital del Instituto Nacional de Estadística y Geografía, en http://cuentame. inegi.org.mx/monografias/informacion/chih/poblacion/m_migratorios.aspx?tema $=$ me\&e $=08$, el 21 de agosto de 2017. 
de los otros casos se refirió que las víctimas fueran originarias de lugar distinto a Ciudad Juárez o el Estado de Chihuahua. Esto no desliga la trata de personas del estatus migrante. Solo indica que en el caso del Estado de Chihuahua, el vínculo es tenue y el problema es fundamentalmente local. La tabla 4.0 muestra el resultado en la búsqueda sobre el lugar de origen de los casos de víctimas que se integran en esta sección dejando ver el aspecto migratorio, al menos en este estudio que se compone de 27 víctimas. Claramente, el estatus migratorio no es una causal de origen ni de fortalecimiento al problema en la forma en que en la actualidad se despliega la actividad de la trata sexual, porque este factor no fue referido en relación con los casos mencionados por ninguno de los colaboradores entrevistados y que actúan como integrantes de la comunidad gubernamental enfocada en atender el problema, ni como resultado de la revisión de expedientes a los que se tuvo acceso. Empero, lo anterior hace necesario matizar la lectura de lo que más adelante en la tabla 4.0 se presenta sobre migración y trata como resultado de los casos que integran este estudio, porque, como ya se indicó, hay bibliografía que sí relaciona el estatus migratorio con la trata. Se puede concluir solo que en el caso del Estado de Chihuahua, este vínculo es relativamente débil. Para el análisis de esta sección se consideraron 29 casos.

En cuanto al lugar de origen revela que 1 víctima es del Estado de Tamaulipas, 1 de Veracruz, 2 víctimas son del municipio de Chihuahua y 25 de Juárez, lo que se determinó de acuerdo con la respuesta obtenida al aplicar las entrevistas para que de ahí se derive si el aspecto migratorio juega un rol causal propiciatorio o fortalecedor en el problema. Sin embargo, las respuestas obtenidas de estos casos concretos reflejan que dicho aspecto no tuvo presencia asociada a la trata sexual, según se desprende de la información que se muestra a continuación, y la tabla 4.0 indica que el problema es fundamentalmente local.

No obstante, de la entrevista con la representante de la congregación religiosa Hermanas Oblatas del Santísimo Redentor, se informa que actualmente hay un grupo de 10 mujeres adultas, originarias de Tlaxcala que fueron deportadas de Estados Unidos de Norteamérica y viven en Ciudad Juárez, ejerciendo la prostitución, de la que deben reportar las ganancias a su explotador que desde Tlaxcala monitorea sus actividades y las tiene sujetas al grado de que cuando ellas tienen que hacer un gasto personal, se comunican telefónicamente con él para que les dé su autorización (comunicación personal, Hermana Nixa, $1^{\circ}$ de marzo de 2017) ${ }^{15}$. Esto indica que aunque sean ya residentes permanentes de Ciudad Juárez, su desplazamiento sí ha

15 Las Hermanas Oblatas del Santísimo Redentor es una congregación religiosa que desde hace diez años realiza trabajo de búsqueda de mujeres en prostitución para ofrecerles otras opciones de vida. Realizan acompañamiento psicológico y de rehabilitación en mujeres que así lo deseen. 
incidido en su vulnerabilidad para ser explotadas sexualmente. En otras palabras, el vínculo entre el desplazamiento físico y la trata debe ser investigado más a fondo.

Tabla 4.0

Migración y trata sexual en Chihuahua

\begin{tabular}{|c|c|c|}
\hline $\begin{array}{l}\text { Fuente de } \\
\text { información }\end{array}$ & $\begin{array}{l}\text { Número } \\
\text { de casos }\end{array}$ & Lugar de origen \\
\hline Arroyo El Navajo & 11 & Juárez \\
\hline \multirow{5}{*}{$\begin{array}{c}\text { Subprocuraduría de protección a N, N y } \\
\text { A, distrito Bravos }\end{array}$} & 1 & Juárez \\
\hline & 1 & Juárez \\
\hline & 1 & Tamaulipas \\
\hline & 1 & Juárez \\
\hline & 1 & Veracruz \\
\hline $\begin{array}{c}\text { Procuraduría de protección a N, N y A } \\
\text { del Estado de Chihuahua }\end{array}$ & 1 & Juárez \\
\hline \multirow{2}{*}{$\begin{array}{c}\text { Fiscalía de atención a víctimas y } \\
\text { ofendidos del delito }\end{array}$} & 1 & Juárez \\
\hline & 1 & Juárez \\
\hline Víctima 1 & 1 & Juárez \\
\hline \multirow{4}{*}{ Víctima 2} & 1 & Juárez \\
\hline & 1 & Juárez \\
\hline & 1 & Juárez \\
\hline & 1 & Juárez \\
\hline Víctima 3 & 1 & Sin información \\
\hline \multirow{2}{*}{ Libres por amor A.C. } & 1 & Chihuahua \\
\hline & 1 & Chihuahua \\
\hline \multirow{2}{*}{ Fiscalía Especializada de la Mujer } & 1 & Juárez \\
\hline & 1 & Juárez \\
\hline
\end{tabular}

Fuente: elaboración propia. 
Aunado a la información obtenida de las entrevistas aplicadas a integrantes de la comunidad gubernamental, víctimas y organismos de la sociedad civil de Ciudad Juárez y Chihuahua, también en las notas de prensa se realizó la búsqueda sobre el factor migratorio y su relación con la trata sexual en el Estado, particularmente en el extremo fronterizo de la región Ciudad Juárez-El Paso, encontrándose una declaración vertida por un agente especial de Homeland Security Investigations de la ciudad de El Paso, Texas, donde este señala que contrariamente a lo que se ha pensado, en esa ciudad la trata de personas es de carácter doméstico porque ciudadanos americanos actúan como tratantes de otros ciudadanos americanos. Es decir, que en la región fronteriza de Ciudad Juárez-El Paso, actualmente los casos se han focalizado con víctimas locales de cada una de las ciudades (El Paso Times, 9 de agosto de 2016) ${ }^{16}$, pero no se encontraron referencias de un movimiento migratorio de adolescentes que sean trasladadas por Juárez, por Puerto Paloma ni por Ojinaga a algún lugar de Estados Unidos de Norteamérica.

Además, durante la búsqueda de insumos para la integración de datos del presente estudio, se entrevistó a un servidor público atendiente de casos en la Fiscalía especial para los delitos de violencia contra las mujeres y trata de personas (FEVIMTRA), delegación Juárez, y a quien se le preguntó específicamente sobre denuncias por este delito en víctimas asociadas a migración nacional e internacional. El entrevistado señaló que a la fecha de la entrevista no existen carpetas de investigación abiertas relativas a este ilícito en referencia al estatus migratorio de las víctimas (comunicación personal, Pedro Vázquez, 2 de octubre de 2016) ${ }^{17}$. En el mismo sentido es la información proporcionada por la directora general adjunta de representaciones temporales de la Comisión Nacional para prevenir y erradicar la violencia contra las mujeres (CONAVIM), oficina de Ciudad Juárez al señalar que

Para sorpresa mía, nos damos cuenta de que ya es un discurso que se ha quedado en la psique colectiva, es decir, todos decimos que hay mucha rotación de población, sin embargo en un estudio del Colegio de la Frontera Norte donde arroja que la gente que permanece en Juárez, ya es de Juárez porque tiene más de 5 años radicando aquí, es decir, ya se quita del imaginario esta creencia de que todos los delitos tienen facilidad de cometerse porque hay gente de muchas otras partes del país (comunicación personal, Zulay Abud Esparza,12 de octubre de 2016).

16 Agents: Human-trafficking victims are from US. Lorena Figueroa, El Paso Times Published, Aug. 9, 2016, Updated 9:57 p.m.

17 En cuanto al nombre del entrevistado, se utiliza para esta investigación con el seudónimo que el servidor público indicó en el consentimiento informado. La entrevista se realizó el domingo 2 de octubre de 2016 en la Biblioteca Central de la Universidad Autónoma de Ciudad Juárez. 
A lo anterior hay que agregar lo propio sobre los 11 casos que integran el llamado juicio histórico de víctimas encontradas sin vida en el Arroyo El Navajo, y de las cuales se encuentra que todas ellas residían en Ciudad Juárez de forma permanente. Ninguna de ellas fue traída de otros estados del país o del extranjero con la intención de ingresarlas a Estados Unidos de Norteamérica. Empero, la información que proporciona la Fiscalía de atención a víctimas y ofendidos del delito zona norte indica que sí han tenido casos de víctimas provenientes de Honduras y Guatemala que han estado sujetas a la explotación sexual (Comunicación personal, 9 de noviembre de 2016) ${ }^{18}$.

Adicionalmente y de acuerdo con la información oficial de la Fiscalía especial para los delitos de violencia contra las mujeres y trata de personas (FEVIMTRA) muestra en su página electrónica que en lo relativo al Estado de Chihuahua durante los años 2008, 2009 y 2011 no se registraron denuncias por trata de personas, mientras que en el año 2010 hubo 7, en 2012 con 9, en 2013 y 2014 hubo dos casos por año, sumando un total de 20 denuncias presentadas en un período de 6 años. La información oficial no refleja datos de 2015, 2016 y $2017^{19}$. Esto implica que dicha agencia de gobierno no maneja datos recientes ni actualizados sobre la dinámica migratoria asociada con la trata de personas, lo que puede revelar que esta instancia no puede incidir adecuadamente en la construcción de políticas públicas con los resultados mostrados.

De cualquier manera, y aun cuando no se cuenta con datos oficiales con respecto al vínculo de migración y trata, se insiste en que la información obtenida durante el levantamiento de trabajo de campo para esta sección, no es contundente en relación con la hipótesis auxiliar $\mathrm{H} 1$ a planteada en cuanto a que la migración es un factor de incidencia para la trata de personas con fines de explotación sexual en mujeres adolescentes de 12 a 18 años. Este hallazgo, sin embargo, no puede ser considerado como final, es decir, que descarte la hipótesis propuesta para esta investigación, precisamente porque se requiere mayor investigación sobre este vínculo. No se puede descartar porque lo que se encontró es referencia de información sobre víctimas procedentes de otros estados del país, pero que no corresponden a casos captados por las agencias de gobierno.

A continuación se presentan las conclusiones que se articulan a partir de los hallazgos discutidos donde se condensan los resultados derivados de la obtención de

18 El servidor público entrevistado en la Fiscalía de atención a víctimas y ofendidos del delito solicitó no ser citado con su nombre, por lo que se omite. La entrevista se realizó en las instalaciones de dicha fiscalía el 9 de noviembre de 2016.

19 Fiscalía especial para los delitos de violencia contra las mujeres y trata de personas. Consultada en http:// www.pgr.gob.mx/Fiscalias/fevimtra/Paginas/default.aspx el 24 de agosto de 2017. 
información y el análisis de los datos obtenidos y contrastados con las hipótesis planteadas para esta sección.

\section{Conclusiones del estudio}

Este trabajo fue orientado para delinear los factores referentes a las víctimas que inciden como aspectos de causa que propician o fortalecen el problema en las adolescentes, obteniendo como resultado lo siguiente. La vulnerabilidad ante la trata sexual sobre los casos que conforman este estudio se integra del siguiente resultado que valida claramente dos de las cuatro hipótesis propuestas y deja una necesidad de mayor investigación. Tal sumatorio de vulnerabilidad se puede enunciar de la siguiente manera:

$\mathrm{H} 1 \mathrm{v}+\mathrm{H} 2 \mathrm{v}=$ vulnerabilidad ante la trata sexual en Chihuahua.

Lo anterior se lee así porque esas dos hipótesis planteadas muestran fuerza explicativa, ya que cuanto más bajo es el nivel socioeconómico, mayor es la susceptibilidad de caer víctimas de trata y a mayor carencia afectiva en el entorno familiar, mayor es el riesgo de ser víctima. El estatus migratorio es más tenue precisamente porque la mayoría de las víctimas son locales.

Un hallazgo importante resulta de la hipótesis $\mathrm{H} 3 \mathrm{v}$ relativa a mayor uso de redes sociales mayor situación de vulnerabilidad para que las adolescentes sean captadas por los tratantes, lo que no integra un patrón respecto de los casos expuestos, sin embargo, el papel de las redes sociales en cuanto al problema de trata en Chihuahua no ha sido documentado en términos de casos judiciales porque no se ha llegado a integrar como tal, pero sí se encuentra que es considerado por la comunidad gubernamental como una fase previa por el alto riesgo que representa el intercambio de fotografías de tono sexual que encuadra en la figura delictiva del sexting, pero no del tipo penal de la trata.

El factor migratorio no se revela en este estudio como aspecto de incidencia ni fortalecedor del problema sobre los casos revisados; pero tampoco es un factor que deba desecharse porque lo mostrado indica que hay casos de víctimas originarias de otras entidades del país que son trasladadas para la trata sexual, sin embargo, no han sido documentadas por las instituciones formales que deben dar cuenta y seguimiento de los casos.

Hasta aquí se exponen los hallazgos y resultados obtenidos sobre las variables incidentes para la victimización en los casos a los que se tuvo acceso de las víctimas de trata sexual en el Estado de Chihuahua, logrando consolidar los datos 
en correlación con las hipótesis propuestas para el estudio. Se dejan como líneas futuras de una investigación con mayor nivel de profundidad lo relativo al estatus migratorio y su incidencia con la trata sexual.

\section{Referencias}

Acharya, A. K. y Salas, A. (2005, septiembre). Violencia y tráfico de mujeres en México: una perspectiva de género. Estudios Feministas. Florianópolis, pp. 507-524. Recuperado de: http://www.scielo.br/pdf/ref/v13n3/a03v13n3

Acharya, A. K. (2016, septiembre). Representation of Human Trafficking in Mexican mass media and its complexity on law enforcement. Journal of Feminist, Gender and Women Studies. Volumen (4), pp. 11-19. Recuperado de: https://revistas.uam.es/revIUEM/article/ view/7283/7655

Azaola, E. y Estes, R. (2003). La infancia como mercancía sexual. México, Canadá, Estados Unidos (primera edición). México D.F.: Siglo XXI Editores, S.A. de C.V.

Casillas, R. (2011). Redes visibles e invisibles en el tráfico y la trata de personas en Chiapas. Migración y Seguridad: nuevo desafío en México, Colectivo de Análisis de la Seguridad con Democracia A.C. (CASEDE). Natalia Armijo (editora). Recuperado de: https://www. casede.org/PublicacionesCasede/MigracionySeguridad/cap3.pdf

Chávez, M. R., Chávez, M. A., RamÍrez, E. y Manríquez, D. (2011). Eficacia de los Instrumentos Internacionales y Nacionales para Erradicar la Explotación Sexual Comercial Infantil en México. En R. Orozco (Ed), Trata de Personas (pp. 25-64). México D.F.: Instituto Nacional de Ciencias Penales. Recuperado de: http://www.inacipe.gob.mx/stories/publicaciones/ temas_selectos/Trata.pdf

Cruz, D. (2013). La trata de personas en agravio de niños, niñas y adolescentes. Lima, Perú: República del Perú, Defensoría del Pueblo. Recuperado de: http://migracion.iniciativa2025alc. org/download/09PEb_Trata_agravio_NNA.pdf

Della, C. (2014, diciembre). La globalización como factor propiciador de la criminalidad organizada transnacional y la trata de personas. ESD. Estudios de Seguridad y Defensa (4), pp. 45-58. Recuperado de: http://esd.anepe.cl/wp-content/uploads/2015/04/ESD04ART02.pdf

Dena, O. (2015). Glosario de términos Jurídico-filosóficos. Curso de filosofía del Derecho. Apoyo Didáctico. Ciudad Juárez: Universidad Autónoma de Ciudad Juárez.

Estévez, D. (2011). Esclavos del siglo XXI. Criminalidad, Trata de personas. pp. 2-6. Recuperado de: http://www.huellasmexicanas.org/alejandra/migracion-y-derechos-humanos/P7\%20 03\%20TRATA\%20PERSONAS.pdf 
Fernández, V. (2006, marzo). Introducción a la Investigación en Ciencias Sociales. Obtenido de https://upcommons.upc.edu/bitstream/handle/2117/501/Introducci\%C3\%B3n\%20a\%20 la\%20investigaci\%C3\%B3n\%20en\%20ciencias\%20sociales.pdf

Gómez, E. y Ontiveros, M. (2004). Estudio Jurídico-Penal Relativo a la Explotación Sexual Comercial Infantil: Bases para su unificación legislativa en México. México D.F.: Organización Internacional del Trabajo.

Negrete, N. (2015). De la "nube" a la internet profunda. La explotación sexual comercial de niñas, niños y adolescentes vinculada a las tecnologías de la información y la comunicación. México D.F.: EDIAC-ECPAT.

Orozco, R. (2011). Trata de Personas. México D.F.: Instituto Nacional de Ciencias Penales.

Rubio, R. (2011). Memoria del Foro Bienal Iberoamericano de Estudios del Desarrollo. Sede: Universidad Autónoma de Ciudad Juárez, México, del 11 al 13 de abril de 2011. Recuperado de: http://riedesarrollo.org/memorias/2011/pdf/M4-2.pdf

UNODC. (2014). Diagnóstico Nacional sobre la Situación de Trata de Personas en México. México D.F.: UNODC. Recuperado el 1 de Febrero de 2015, del sitio: http://www.unodc.org/documents/mexicoandcentralamerica/Diagnostico_trata_de_personas.pdf

Recibido: 22/10/2017

Aceptado: $14 / 3 / 2018$ 